\title{
In-situ observation of gas hydrate formation using a transparent high pressure cell as a simulation of the bottom of ice crust in icy bodies
}

\author{
ATSUSHI TANI, KENSHIRO MORIYAMA AND HANAE \\ OZAKI
}

Kobe University

Presenting Author: tani@carp.kobe-u.ac.jp

Gas hydrates are clathrate compounds where hydrogen-bonded water cages include a guest gas molecule. Methane hydrate is one of famous gas hydrates because it is naturally found in deepsea and permafrost sediments on the Earth. Gas hydrates would exist in icy bodies as well. One of the candidates is Pluto where the presence of subsurface ocean is suggested. Gas hydrates may exist between the ice shell and subsurface ocean, and play an important role as insulator to keep warm inside. If granular gas hydrates exist in the upper part of the subsurface ocean, heat can be transferred by not only thermal conduction but also convection. This means that icy bodies may be cooled faster. To elucidate how gas hydrates form in the subsurface ocean in icy bodies, we developed the in-situ observation system of gas hydrate formation and dissociation under high pressure as a simulation of the bottom of the ice crust.

The observation system was filled with water and set in thermostatic cooling bath. In this study, CF4 gas was supplied to the cell up to $7 \mathrm{MPa}$ to form $\mathrm{CF} 4$ hydrate. The reason why we chose CF4 instead of methane (CH4) is that CF4 hydrate is heavier than water and should sink in the water cell even if the hydrate forms in the water.

Long period observation revealed that 1) at first, tiny hydrate particles formed in the water and drifted in the water after shaking the cell, 2) then, a few $\mathrm{mm}$ size of hydrate crystals formed and stuck on the ice sheet, 3) hydrate particles were embedded in the ice if formation rate of ice was faster than that of the hydrate. These suggest that 1) gas hydrate layer beneath the ice sheet in icy bodies may form after the growth of the ice sheet becomes slow and 2) ice and gas hydrate mixed layer may be formed above the gas hydrate layer. 